\title{
The microbiology of chronic osteomyelitis in a developing world setting
}

\author{
PG Mthethwa MBBCh, H Diploma(Ortho)SA \\ University of KwaZulu-Natal \\ LC Marais MBChB, FC(Ortho)SA, MMed(Ortho), CIME, PhD \\ School of Clinical Medicine, University of KwaZulu-Natal \\ Corresponding author: \\ Dr Phakamani G Mthethwa \\ Tumour, Sepsis and Reconstruction Unit \\ Department of Orthopaedic Surgery \\ Grey's Hospital \\ Nelson R. Mandela School of Medicine \\ University of KwaZulu-Natal \\ Pietermaritzburg, South Africa \\ Tel: +27338973000 \\ Email: phakspg@gmail.com
}

Registrar, Department of Orthopaedics, Grey's Hospital, School of Clinical Medicine,

Tumour, Sepsis and Reconstruction Unit, Department of Orthopaedics, Grey's Hospital,

\begin{abstract}
Objectives: The primary aim of this study was to identify the microorganisms that cause chronic osteomyelitis in a developing world clinical setting and to characterise the antibiotic sensitivity profile of these pathogens. Furthermore, we aimed to determine whether the causative organisms vary in relation to physiological status of the host, the HIV status of the patient or the cause of the infection (post-traumatic, post-operative and haematogenous).

Methods: We performed a retrospective review of consecutive adult patients treated curatively for chronic osteomyelitis of long bones, over a two-year period. Patient charts were reviewed and data extracted in respect of patient demographics, the cause of infection, physiological status of the host in accordance with the Cierny and Mader classification, HIV status, surgical treatment strategy and causative organism.

Results: A total of 108 organisms were identified in the 60 patients included in the study. Multiple organism were cultures in $45 \%$ of patients, a single Gram-positive organism in $22 \%$ and a single Gram-negative organism in $26 \%$ of patients. In four cases $(7 \%)$ no causative organism was cultured. The most prevalent organisms were Enterobacteriaceae (34\%), Staphylococcus spp. (29\%), Pseudomonas aeruginosa (11\%), and Enterococcus spp. (9\%). Many isolates were found to be resistant to commonly used empirical anti-microbial agents. Seventy per cent of Enterobacteriaceae spp. were resistant to either cefuroxime and/or ampicillin-clavulanic acid. Seventy-seven per cent of Staphylococcus aureus isolates were susceptible to cloxacillin. More than 50\% of Pseudomonas aeruginosa strains were resistant to meropenem, imipenem, piperacillin-tazobactam or cefepime. There was a significant association between the aetiology of the infection and the microorganisms involved ( $p$-value $<0.01$ ). The bacterial pathogen profile was, however, not associated with the physiological status of the host $(\mathrm{p}=0.22)$ or the HIV status of the patient.

Conclusion: While the majority of haematogenous chronic osteomyelitis still involved a solitary Gram-positive organism, the incidence of Gram-negative infections was found to be higher than previously reported. Contiguous chronic osteomyelitis was mostly polymicrobial in nature and solitary infections involving a Gramnegative organism was most common in the post-traumatic group. The bacterial pathogen did not vary in relation to the HIV status of the patient or the physiological status of the host.
\end{abstract}

Key words: chronic, osteomyelitis, microbiology, antibiotics, treatment, HIV 


\section{Introduction}

The diagnosis of chronic osteomyelitis encompasses a range of bone infections resulting from either haematogenous bacterial seeding or direct spread from a contiguous focus. ${ }^{1}$ Traditionally, the clinical entity of chronic osteomyelitis has been dominated by haematogenous disease. This form of the disease is typically associated with a narrow range of causative organisms. ${ }^{1}$ In recent times this category has been supplemented considerably by contiguous infections which are caused by a much wider range of microorganisms. ${ }^{23}$ Posttraumatic, post-operative and implant-related infections are now encountered much more frequently, especially in developed regions. ${ }^{2,3}$ Overall, infectious complications can be expected in approximately $1-5 \%$ of internally fixated closed fractures and in up to $30 \%$ of open fractures. ${ }^{4}$ Irrespective of the cause, chronic osteomyelitis remains difficult to treat and treatment failure may occur in more than $10 \%$ of cases. ${ }^{4}$

Treatment options for chronic osteomyelitis include curative and palliative management strategies. ${ }^{5,6}$ Curative treatment protocols involve a combination of thorough debridement, dead space management, soft tissue reconstruction and adjuvant antibiotic therapy in order to achieve remission of the disease prior to reconstruction. Palliative treatment strategies, may be indicated in compromised hosts who are not suitable for complex surgery., 5 Typically palliative treatment involves chronic suppressive antibiotic therapy. Knowledge of the microbiology of chronic osteomyelitis may guide the selection of empiric postoperative or palliative antibiotic therapy.

There is limited data available on the spectrum of causative organisms, especially in contiguous chronic osteomyelitis. Previous studies have shown that the most common causative organism remains Staphylococcus aureus, followed by Enterobacteriaceae. ${ }^{1-3}$ Few studies have been performed in the developing world. Furthermore, the variation of causative organism in relation to the physiological host status, the HIV status of the patient or the cause of the infection has not previously been reported.

The primary aim of this study was to identify the microorganisms that cause chronic osteomyelitis in the developing world clinical setting. In addition, we aimed to characterise the antibiotic sensitivity profile of these pathogens. Furthermore, we aimed to determine whether the causative organisms vary in relation to the physiological status of the host, the HIV status of the patient or the cause of the infection (post-traumatic, post-operative and haematogenous).

\section{Methods}

We retrospectively reviewed a cohort of consecutive patients who underwent surgical treatment of chronic osteomyelitis at our tertiary level musculoskeletal tumour and sepsis unit over the two-year period from January 2012 to December 2013. Following ethical approval, eligible patients were identified from a prospectively gathered database. All patients, 14 years or older, who underwent surgical treatment for chronic osteomyelitis were included in the study. Cases involving tuberculosis or fungal infection, as well as cases with insufficient data, were excluded from the study.

The patients' charts and X-rays were reviewed and data extracted in respect of patient demographics, the cause of infection (haematogenous or contiguous) and the host classification in accordance with the Cierny and Mader classification system. ${ }^{7}$ The patient's HIV status, the surgical treatment strategy employed and the causative organism, along with their antibiotic sensitivity profiles, were also recorded.

In all cases, formal tissue biopsy for the purposes of histology and microscopy, culture and sensitivity testing was performed at the time of the initial debridement. Antibiotic treatment was discontinued at least seven days prior to tissue sampling. Samples were processed by agitating them in a sterile glass ball in $10 \mathrm{ml}$ of Peptone water under laminar flow. Samples were then plated onto blood agar (incubated aerobically and anaerobically) and lysed blood agar (incubated in air with 5\% CO2). Extended culture, for a minimum period of seven days, was routinely performed. Organisms' antimicrobial sensitivity was determined by VITEK2 technology which identifies the vast majority of routine organisms (over 300 microorganisms).

For the purposes of this study chronic osteomyelitis was defined as a bone infection characterised by the presence of either necrotic bone or an implant which may serve as a nidus for biofilm formation and a duration of at least 6 weeks. ${ }^{8}$ Contiguous chronic osteomyelitis was defined as post-traumatic (following open fractures) and postoperative infections, as well as bone infection resulting from direct spread from an adjacent septic focus or ulceration. ${ }^{9}$

Statistical analysis was performed using Stata 13.0 (Stata Corp. College Station, Texas). Continuous variables were reported as mean $( \pm S D)$ or median (with interquartile range) and categorical variables as number and percentages, unless otherwise stated. The Fisher's exact test was used for categorical data. All tests were two-sided and the level of significance was set at $\mathrm{p}<0.05$.

Ethical approval was obtained from our institution's ethics review board prior to commencement of the study (BREC: BE020/14). The study was conducted according to the ethical principles for medical research on human subjects as defined by the World Medical Association Declaration of Helsinki (amended at the MA General Assembly, Seoul, Oct 2008).

\section{Results}

A total of 60 patients, from the 64 initially identified to meet the inclusion criteria, were included in this cohort. Of the four patients who were excluded, one was diagnosed with tuberculosis osteomyelitis and one with a fungal infection (Cryptococcus neoformans), and two cases were excluded on the basis of insufficient data. The mean age of the remaining 60 patients was 31 years (range 14-59 years), with 47 males and 13 females. 
A total number of 108 microorganisms were identified and multiple organisms were cultured in $45 \%(\mathrm{n}=27)$ of patients, a single Gram-positive organism in $22 \%$ and a single Gramnegative organism in $26 \%$ of patients. In four cases (7\%) no causative organism was cultured. The most common causative organisms was found to be from the Enterobacteriaceae group (Citrobacter, Enterobacter, Escherichia, Klebsiella, Morganella, Pantoea, Proteus and Serratia spp.; $34 \%, \mathrm{n}=37)$, followed by Staphylococcus spp. (29\%, $\mathrm{n}=31)$, Pseudomonas aeruginosa $(11 \%, \mathrm{n}=12)$ and Enterococcus spp. $(9 \%, \mathrm{n}=10)$ (Table I). In terms of individual organisms, $S$. aureus was the most common isolate $(\mathrm{n}=23)$.

Seventy per cent of Enterobacteriaceae were resistant to either cefuroxime and/or ampicillin-clavulanic acid. Resistance to piperacillin-tazobactam and cefepime was observed in $29 \%$ and $34 \%$ of Enterobacteriaceae cultures, respectively. Enterobacteriaceae sensitivity to ciprofloxacin, gentamycin and meropenem was $71 \%, 81 \%$ and $89 \%$ respectively.

Table I: Microorganisms cultured in the cohort of 60 patients

Multiple organisms were identified in $27(46 \%)$ of cases.

\begin{tabular}{|l|c|}
\hline Microorganism & $\mathbf{n}(\%)$ \\
\hline Acinetobacter baumannii & $7(6 \%)$ \\
\hline Aeromonas hydrophylia & $1(1 \%)$ \\
\hline Citrobacter amalonaticus & $1(1 \%)$ \\
\hline Citrobacter koseri & $1(1 \%)$ \\
\hline Escherichia coli & $5(5 \%)$ \\
\hline Entecoccus faecalis & $1(1 \%)$ \\
\hline Enterobacter cloacae & $6(6 \%)$ \\
\hline Enterobacter species & $1(1 \%)$ \\
\hline Enterococcus avium & $1(1 \%)$ \\
\hline Enterococcus faecalis & $7(6 \%)$ \\
\hline Enterococcus faecalis & $1(1 \%)$ \\
\hline Klebsiella oxytoca & $2(2 \%)$ \\
\hline Klebsiella pneumonia & $5(5 \%)$ \\
\hline Lactococcus lactis subsp lactis & $1(1 \%)$ \\
\hline Methicillin-resistant Staphylococcus aureus & $5(5 \%)$ \\
\hline Methicillin-sensitive Staphylococcus aureus & $19(18 \%)$ \\
\hline Morganella morganii subsp sibonii & $2(2 \%)$ \\
\hline No growth & $4(4 \%)$ \\
\hline Proteus maribilis & $7(6 \%)$ \\
\hline Pseudomonas aeruginosa & $12(11 \%)$ \\
\hline Serratia marcescens & $7(6 \%)$ \\
\hline Staphylococcus capitis & $2(2 \%)$ \\
\hline Staphylococcus epidermidis & $2(2 \%)$ \\
\hline Staphylococcus haemolyticus & $1(1 \%)$ \\
\hline Staphylococcus hominis4 & $1(1 \%)$ \\
\hline Staphylococcus simulans & $1(1 \%)$ \\
\hline Streptococcus mitis & $1(1 \%)$ \\
\hline Streptococcus pneumoniae & $1(1 \%)$ \\
\hline Streptococcus pyogenes & $2(2 \%)$ \\
\hline Streptococcus sanguinis & $1(1 \%)$ \\
\hline Total & $108(100 \%)$ \\
\hline
\end{tabular}

Fourteen $(77 \%)$ of Staphylococcus aureus isolates were susceptible to cloxacillin. S. aureus exhibited a similar susceptibility pattern to other common anti-staphylococcal agents, including erythromycin $(84 \%)$, clindamycin $(84 \%)$ and trimethoprim-sulfamethoxazole $(68 \%)$. Five $(23 \%)$ of the S. aureus cultures and six $(86 \%)$ of the other Staphylococcus spp. were found to be methicillin-resistant. None of the methicillin-resistant $S$. aureus strains were resistant to vancomycin. We noted a high rate of resistance $(\geq 50 \%)$ of Pseudomonas aeruginosa strains to meropenem, imipenem, piperacillin-tazobactam or cefepime. More than $80 \%$ of $P$. aeruginosa strains were sensitive to gentamycin and ciprofloxacin. Fifty per cent of Enterococcus spp. were ampicillin-resistant. No vancomycin-resistant Enterococcus spp. were cultured (Table II).

The cause of chronic osteomyelitis was haematogenous in $28 \%(n=17)$ of cases. Seventy-two per cent $(n=43)$ of cases were classified as contiguous, with post-operative and post-traumatic infection comprising $17 \%(\mathrm{n}=10)$ and $55 \%(\mathrm{n}=33)$ of all cases, respectively. There was a significant association between the origin of the infection and the microorganism involved ( $\mathrm{p}$-value $=0.003)$. The majority of contiguous infections involved mixed organisms, while solitary infections by a Gram-negative organism were most common in the post-traumatic group (Table III). Twenty-one organisms were cultured in the 10 patients with post-operative infection. The most common pathogens were Acinetobacter baumannii $(\mathrm{n}=5)$, Enterobacter spp. $(\mathrm{n}=5)$, E. coli $(\mathrm{n}=4)$ and Enterococci $(\mathrm{n}=3)$. Sixty-two organisms were found in the post-traumatic group of 33 patients. The most common pathogens in this group was methicillin-sensitive Staphyloccus aureus $(n=7)$, Serratia marcescens $(n=7)$, Pseudomonas aeruginosa $(n=6)$, Acinetobacter baumannii, Enterobacter spp., Proteus maribillis, Klebsiella spp. $(\mathrm{n}=4$, respectively), E. coli and MRSA $(\mathrm{n}=3)$, respectively. In the haematogenous group of infections, the majority of infections were caused by a solitary Grampositive organism. Methicillin-sensitive Staphylococcus aureus was cultured in nine out of the 17 haematogenous cases, although only four of these were monomicrobial in nature. Methicillin-resistant Staphyloccus aureus and Pseudomonas spp. were responsible for two cases respectively. Four $(24 \%)$ of haematogenous cases were polymicrobial in nature.

When applying the Cierny and Mader classification system $25 \%(\mathrm{n}=15)$ patients were classified as A-hosts, 55\% $(\mathrm{n}=33)$ as B-hosts and $20 \%(\mathrm{n}=12)$ as C-hosts. ${ }^{10}$ Six type-A hosts had Gram-positive infections, while only three had mixed infections. The majority of infections (70\%) in B and $\mathrm{C}$ hosts were caused by multiple organisms. This difference was, however, not statistically significant $(\mathrm{p}=0.22)$ (Table IV).

Eleven patients (18\%) were found to be HIV-positive, with a median CD4 count of 544 cells $/ \mathrm{mm}^{3}$ (range 194-1034). No significant difference was noted in terms of Gram-positive, Gram-negative, mixed pathogen or no growth cultures 


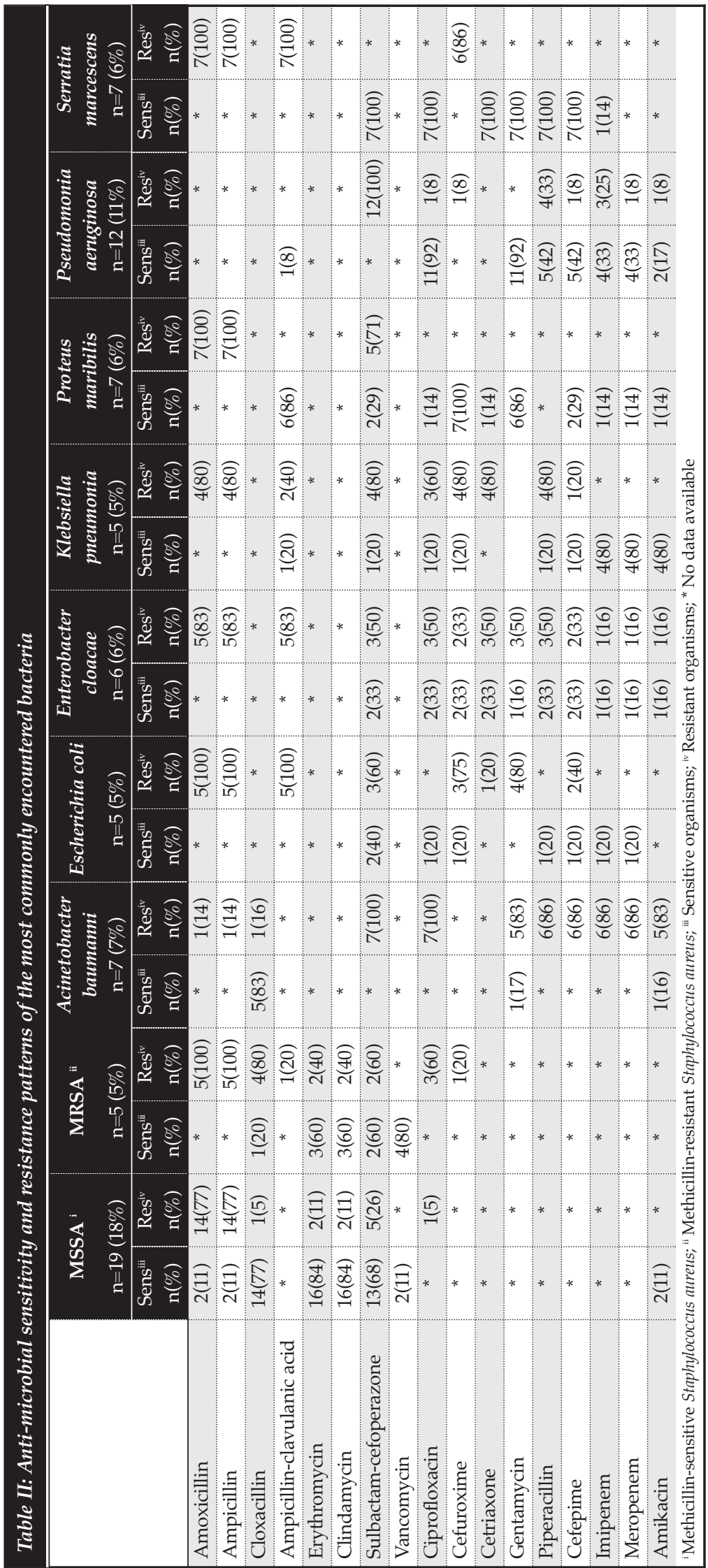

between HIV-positive and -negative patients $(\mathrm{p}=0.18)$ (Table V). Furthermore, when comparing individual pathogens in HIV-positive and -negative patients, no significant differences were found. While there appeared to be an increased incidence of Enterococcus spp. infections in HIV-positive patients, it was not statistically significant $(\mathrm{p}=0.18)$.

\section{Discussion}

The aim of this study was to characterise the organisms causing chronic osteomyelitis in a developing world setting and to compare it to existing data from other parts of the world. Furthermore, we examined the anti-microbial resistance and sensitivity patterns. We also aimed to determine if the pathogen profile varied in relation to the origin of the infection, the physiological status of the host or the patient's HIV status. While the series is small, a wide spectrum of organisms with a wide variety of antibiotic sensitivities was found. This emphasises the importance of identifying the offending bacteria by tissue culture, and tailoring therapy according to antibiotic sensitivity patterns.

Methicillin-sensitive Staphylococcus aureus was the most commonly isolated Gram-positive organism (18\% of all organisms), followed by other Staphylococcal species (7\%), Enterococcus $(6 \%)$, methicillin-resistant Staphylococcus aureus (5\%) and Streptococcus spp. (4\%). The most common Gramnegative organism was Pseudomonas aeruginosa (11\% of all organisms), followed by Enterobacter, Acinetobacter, Serratia and Proteus spp. (6\%, each). Our results are comparable to previous studies from other parts of the world (Table VI). Walvogel et al., in 1970, found Staphylococcus aureus in approximately $60 \%$ of cases. ${ }^{1}$ In subsequent studies the relative incidence of staphylococcal infections decreased, possibly due to a rise in the incidence of contiguous infections. ${ }^{2}$ Post-operative or post-traumatic chronic osteomyelitis has been associated with broad spectrum of organisms, including those commonly associated with hospital acquired infections like methicillinresistant S. aureus, enteric Gram-negative bacilli and coagulase-negative Staphylococci., ${ }^{2,3}$ However, S. aureus appears to remain the most commonly isolated organism, regardless of aetiology (Table VI). Trampuz et al. identified Staphylococcus aureus (30\% of cases) and Gram-negative micro-organisms as the most common bacteria involved in infections associated with fracture-fixation devices. ${ }^{2}$ Sheehy et al. found Staphylococcus aureus to be the most commonly isolated pathogen among a wide range of organisms, which included Gram-negative bacilli, anaerobes and coagulase negative staphylococci. ${ }^{3}$ 


\begin{tabular}{|c|c|c|c|c|c|}
\hline Aetiology & $\begin{array}{c}\text { Gram-positive } \\
\text { n (\%) }\end{array}$ & $\begin{array}{c}\text { Gram-negative } \\
\text { n }(\%)\end{array}$ & $\begin{array}{l}\text { Mixed } \\
\text { n (\%) }\end{array}$ & $\begin{array}{c}\text { No growth } \\
\text { n }(\%)\end{array}$ & p-value ${ }^{i}$ \\
\hline Post-operative $\mathrm{e}^{\mathrm{ii}}$ & $2(1.8 \%)$ & $2(1.8 \%)$ & $5(4.6 \%)$ & $1(0.9 \%)$ & \multirow{4}{*}{0.003} \\
\hline Post-traumatici & $2(1.8 \%)$ & $12(11.1 \%)$ & $18(16.7 \%)$ & $1(0.9 \%)$ & \\
\hline Haematogenous & $9(8.3 \%)$ & $2(1.8 \%)$ & $4(3.7 \%)$ & $2(1.8 \%)$ & \\
\hline Totals & $13(12.0 \%)$ & $16(14.7 \%)$ & $27(25 \%)$ & $4(3.6 \%)$ & \\
\hline
\end{tabular}

\begin{tabular}{|c|c|c|c|c|c|}
\hline Host status & $\begin{array}{c}\text { Gram-positive } \\
\text { n (\%) }\end{array}$ & $\begin{array}{c}\text { Gram-negative } \\
\text { n (\%) }\end{array}$ & $\begin{array}{c}\text { Mixed } \\
\text { n (\%) }\end{array}$ & $\begin{array}{l}\text { No growth } \\
\text { n }(\%)\end{array}$ & p-value ${ }^{i}$ \\
\hline A-host & $6(10 \%)$ & $4(7 \%)$ & $3(7 \%)$ & $1(2 \%)$ & \multirow{4}{*}{0.221} \\
\hline B-host & $6(10 \%)$ & $10(17 \%)$ & $16(17 \%)$ & $1(2 \%)$ & \\
\hline C-host & $1(2 \%)$ & $2(3 \%)$ & $7(12 \%)$ & $2(3 \%)$ & \\
\hline Totals & $13(22 \%)$ & $16(27 \%)$ & $26(43 \%)$ & $4(7 \%)$ & \\
\hline
\end{tabular}

Table V: Bacterial pathogens in relation to HIV status of the patient

\begin{tabular}{|c|c|c|c|c|c|}
\hline HIV status & $\begin{array}{c}\text { Gram-positive } \\
\text { n }(\%)\end{array}$ & $\begin{array}{c}\text { Gram-negative } \\
\text { n ( } \%)\end{array}$ & $\begin{array}{l}\text { Mixed } \\
\text { n (\%) }\end{array}$ & $\begin{array}{c}\text { No growth } \\
\text { n }(\%)\end{array}$ & p-value \\
\hline Negative & $13(22 \%)$ & $13(22 \%)$ & $20(33 \%)$ & $3(5 \%)$ & \multirow{3}{*}{0.183} \\
\hline Positive & $0(0 \%)$ & $3(5 \%)$ & $7(12 \%)$ & $1(2 \%)$ & \\
\hline Totals & $13(22 \%)$ & $16(27 \%)$ & $27(45 \%)$ & $4(7 \%)$ & \\
\hline
\end{tabular}

\begin{tabular}{l} 
Table VI: Organisms isolated in cases of adult chronic osteomyelitis by previous investigators \\
\hline \\
\hline Study
\end{tabular}

In our series, the majority of post-operative and posttraumatic infections involved Gram-negative or mixed infections.

We found a significant association between the aetiology of the infection (post-traumatic, post-operative or haematogenous) and the causative organism ( $p$-value $=$ 0.003). Haematogenous infections were mostly due to Gram-positive organisms, while the majority of mixed infections occurred in contiguous chronic osteomyelitis. Isolated Gram-negative infections were most common in the post-traumatic group of cases. In this series, the prevalence of polymicrobial infections was somewhat higher $(45 \%)$ than previously reported in studies performed in the developed world setting. ${ }^{2,3}$ Trampuz et al. found polymicrobial infections in $27 \%$ of patients. ${ }^{2}$ Sheehy et al. reported that $29 \%$ of their cases were polymicrobial in nature. $^{3}$

Spellberg and Lipsky reviewed publications, from 1970 to 2011, related to the use of antibiotics in chronic osteomyelitis. ${ }^{11}$ The authors emphasised the importance of surgical debridement and noted that the cure rate of chronic osteomyelitis is increased with surgical resection of infected and devitalised tissue in conjunction with antibiotic therapy. The important conclusions from their review were that oral antibiotic therapy with agents that have high bioavailability is comparable with parenteral therapy; improved cure rates are achieved with the addition of rifampicin; and that the duration of antibiotic therapy should be individualised based on clinical, haematological, and radiological response. ${ }^{11}$ The authors also recommended that antibiotic therapy should be tailored to local sensitivity patterns. The organisms cultured in this series exhibited a wide range of antibiotic sensitivities. Many commonly employed generic antibiotic regimens may be ineffective in our setting. It is alarming that $70 \%$ of the Enterobacteriaceae spp. in this series were resistant to either cefuroxime or ampicillin-clavulanic acid and $34 \%$ of these organisms were resistant to cefepime. 
Our findings conflict with previous data showing that cephalosporins generally have a good activity against commonly isolated enterobacteriaceae. ${ }^{12}$ In our series, Enterobacteriaceae spp. exhibited reasonable sensitivity to ciprofloxacin $(71 \%)$, gentamycin $(81 \%)$ and meropenem $(89 \%)$. This is consistent with the findings of Spellberg et al. who found a high cure rate with a combination use of ciprofloxacin and trimethoprimsulfamethoxazole when given for for $8-16$ weeks. ${ }^{11}$ In our series, $77 \%$ of Staphylococcus aureus were susceptible to cloxacillin. Jones et al. reported similar results, with more than $99 \%$ S. aureus susceptible to oxacillin. ${ }^{12}$ Methicillin-resistant $S$. aureus was however common in our series, with $80 \%$ of strains being susceptible to vancomycin. Vancomycin penetrates bone relatively poorly, with the ratio of bone to serum concentration being only approximately $20 \%$ in infected bone. ${ }^{13}$ Cefazolin outperformed vancomycin in one series, despite its bone penetration being inferior to that of vancomycin (approximately 15\%). ${ }^{14}$ Meropenem does not appear to fare much better, with a bone penetration of approximately $17 \% .{ }^{15}$ Intravenous quinolone antibiotics are another option for Gram-negative cover and exhibit excellent bone penetration. The bone/serum ratio of intravenous ciprofloxacin has been reported to be up to $47 \%$ in cortical bone. ${ }^{16}$ Conveniently, the bone penetration of oral ciprofloxacin is also reasonable, reaching $35 \%$ at a dose of $500 \mathrm{mg} .{ }^{17}$ Taking these results into account the generic antibiotic regimen (for use following surgery) proposed by McNally, involving the use parenteral vancomycin and meropenem, appears to be reasonable. ${ }^{18}$ Ciprofloxacin (or another quinolone) appears to be a useful alternative to meropenem for aerobic Gram-negative bacilli, and previous clinical studies support its use. ${ }^{19}$ Cefepime appears to remain an excellent option in Gram-negative infections due to relatively low resistance and excellent bone penetration. ${ }^{20}$

In our series, the bacterial pathogen profile did not vary in relation to the patient's HIV status. Previous literature identified Staphylococcus aureus as the most common organism causing osteoarticular infections in HIV patients. ${ }^{10,21}$ Zalavras et al. looked at the microbiology of chronic osteomyelitis in patients living with the human immunodeficiency virus and found $S$. aureus in $50 \%$ of cases, polymicrobial infections in $35 \%$ and no growth in three cases. ${ }^{21}$ They also found no osseous opportunistic infection in HIV patients with immunodeficiency. Busch et al. also reported Staphylococcus aureus (in 8 of 19 patients) as the most common agent in HIVinfected patients with chronic osteomyelitis. ${ }^{10}$ Staphylococcus aureus was also shown to be the most common organism responsible for osteoarticular infections in intravenous drug abusers with immunodeficiency virus. ${ }^{22}$ Information about the causative agents was available in $88(27 \%)$ of the 330 osteomyelitis cases identified by the Adult/Adolescent Spectrum of HIV
Disease project of the Centers for Disease Control (CDC). Staphylococcus aureus was the most common pathogen, comprising $48 \%$ of identified organisms. In our study, the majority of HIV-positive patients presented with polymicrobial infections and there was no association between HIV status and causative bacteria ( $\mathrm{p}$-value $=0.18$ ). Therefore, in our series, HIV infection does not appear to alter the microbiology of chronic osteomyelitis. There is insufficient evidence to suggest that clinicians should change their antibiotic protocol based on the HIV status of their patients, and according to the available data it does not appear necessary to add routine antibiotic coverage for opportunistic infections in HIV patients.

Previous studies did not stratify infective organisms in relation to the Cierny and Mader host status or anatomical nature of the disease. In our study shows that there is no significant association between host status (A, B and C-host) and the causative organism ( $p$-value $=0.221)$. Although it did not reach statistical significance, type C-hosts were found to have predominantly polymicrobial infections.

The small number of cases in this series is a shortcoming and much larger studies are required before resolute recommendations can be made in terms of generic antibiotic selection. The absence of routine anaerobe cultures is a further shortcoming. In larger series, anaerobic infections have accounted for a small, but significant, number of infections. An additional shortcoming is the absence of data relating to prior antibiotic therapy and number of previous surgical attempts. Prior treatment may influence the bacterial profile and thus the cultured organism may not necessarily be the original causative organism. Comparative studies are required to determine the clinical efficacy of different antibiotic regimens.

\section{Conclusion}

The causative microorganism in chronic osteomyelitis was found to be associated with aetiology of the infection. While the majority of haematogenous chronic osteomyelitis still involved a solitary Gram-positive organism, the incidence of Gram-negative infections was found to be higher than previously reported. Contiguous chronic osteomyelitis was mostly polymicrobial in nature and solitary infections involving a Gram-negative organism was most common in the post-traumatic group. The bacterial pathogen did not vary in relation to the HIV status of the patient or the physiological status of the host.

\section{Compliance with ethics guidelines}

Conflict of interest: The authors declare that they have no conflict of interests and that no financial support was received for this study. 
Ethics approval: Ethical approval was obtained from our institution's ethics review board prior to commencement of the study (BREC: BE020/14). The study was conducted according to the ethical principles for medical research on human subjects as defined by the World Medical Association Declaration of Helsinki (amended at the MA General Assembly, Seoul, Oct 2008).

Authors' contributions: All authors made contributions toward the conception and design of the research, acquisition of data and drafting of the manuscript. The final manuscript was read and approved by all the authors.

\section{References}

1. Lew DP, Waldvogel FA. Current concepts osteomyelitis. $N$ Eng J Med 1997;336:999-1007.

2. Trampuz A, Zimmerli W. Diagnosis and treatment of infections associated with fracture- fixation devices. Injury 2006;37(Suppl 2):59-66.

3. Sheehy SH, Atkins BA, Bejon P, Byren I, Wyllie D, Athanasou NA, Berendt AR, McNally MA. The microbiology of chronic osteomyelitis: Prevalence of resistance to common empirical anti-microbial regimens. J Infection 2010;60:338-43.

4. Gustilo RB, Merkow RL, Templeton D. The management of open fractures. J Bone Joint Surg Am 1990;72(2);299-304.

5. Walter G, Kemmerer M, Kappler C, Hoffmann R. Treatment Algorithms for Chronic Osteomyelitis. Dtsch Arztebl Int 2012;109(14):257-64.

6. Marais LC, Ferreira N, Sartorius B, Aldous C, Le Roux TLB. A modified staging system for chronic osteomyelitis. J Orthop 2015;12:184-92.

7. Cierny G, Mader JT, Penninck JJ. A clinical staging system for adult Osteomyelitis. Clin Orthop Relat Res 2003;414:724.

8. Cierny G. Surgical treatment of osteomyelitis. Plast Reconstr Surg 2011;127(Suppl1):190-204.

9. Lew DP, Waldvogel FA. Osteomyelitis. Lancet 2004;364: 369-79.

10. Busch V, Regez R, Heere B, Willems J. Osteoarticular infections in HIV-infected patients: 23 cases among 1,515 HIVinfected patients. Acta Orthop 2009;78(6):786-90.

11. Spellberg B, Lipsky A. Systemic antibiotic therapy for chronic osteomyelitis in adults clinical infectious diseases. Clin Infect Dis 2012;54(3):393-407.
12. Jones ME, Karlowsky JA, Draghi DC. Antibiotic susceptibility of bacteria most commonly isolated from bone related infections: the role of cephalosporins in antimicrobial therapy. Int J Antimicrob Agents 2004;23:240-46.

13. Graziani AL, Lawson LA, Gibson GA, Steinberg MA, MacGregor RR. Vancomycin concentrations in infected and non-infected human bone. Antimicrob Agents chemother 1988;32:1320-22.

14. Williams DN, Gustilo RB, Beverly R, Kind AC. Bone and serum concentrations of five cephalosporin drugs. Relevance to prophylaxis and treatment in orthopedic surgery. Clin Orthop 1983;179:253-65.

15. Sano $T$, Sakurai $M$, Dohi $S$, et al. Investigation of meropenem levels in the human bone marrow blood, bone, joint fluid and joint tissues. Jpn J Antibiot 1993;46:159-63.

16. Meissner A, Borner K. Concentration of ciprofloxacin in bone tissue. Aktuelle Traumatol 1993;23:80-84.

17. Fong IW, Ledbetter WH, Vandenbroucke AC, Simbul M, Rahm V. Ciprofloxacin concentrations in bone and muscle after oral dosing. Antimicrob Agents Chemother 1986;29:405408.

18. McNally M, Nagarajah K. Osteomyelitis. Orthop Trauma 2010;24(6):416-429.

19. Gilbert DN, Tice AD, Marsh PK, Craven PC, Preheim LC. Am J Med 1987;82(4A):254-58.

20. Breilh D, Boselli E, Bel JC, Chassard D, Saux MC, Allaouchiche B. Diffusion of cefepime into cancellous and cortical bone tissue. J Chemother 2003;15:134-38.

21. Zalavras C, Gupta N, Patzakis M, Holtom P. Microbiology of osteomyelitis in patients infected with the human immunodeficiency virus. Clin Orthop Relat Res 2005;439;97-100.

22. Belzunegui J, Gonzalez C, Lopez L, Osteoarticular and muscle infections lesions in patients with the human immunodeficiency virus. Clin Rheum 1997;16(5):450-53.

This article is also available online on the SAOA website (www.saoa.org.za) and the SciELO website (www.scielo.org.za). Follow the directions on the Contents page of this journal to access it. 